\title{
High-resolution genome-wide allelotype analysis identifies loss of chromosome 14q as a recurrent genetic alteration in astrocytic
} tumours

\author{
J Hu', JC-s Pang², CY-k Tong'², B Lau'², X-I Yin², W-S Poon ${ }^{3}$, C-C Jiang', L-F Zhou' and H-K Ng*,2 \\ 'Department of Neurosurgery, Hua Shan Hospital, Shanghai Medical University, Shanghai, China; ${ }^{2}$ Department of Anatomical \& Cellular Pathology, Prince of \\ Wales Hospital, The Chinese University of Hong Kong, Hong Kong, China; ${ }^{3}$ Neurosurgical Unit, Department of Surgery, Prince of Wales Hospital, The \\ Chinese University of Hong Kong, Hong Kong, China
}

Diffusely infiltrative astrocytic tumours are the most common neoplasms in the human brain. To localise putative tumour suppressor loci that are involved in low-grade astrocytomas, we performed high-resolution genome-wide allelotype analysis on I7 fibrillary astrocytomas. Non-random allelic losses were identified on chromosomal arms IOp (29\%), 10q (29\%), 14q (35\%), $17 p(53 \%)$, and 19q (29\%), with their respective common regions of deletions delineated at 10p /4-15.I, 10q25.I-qter, 14q212.2-qer, 17p|1.2-pter and 19q12-13.4. These results suggest that alterations of these chromosomal regions play important roles in the development of astrocytoma. We also allelotyped 21 de novo glioblastoma multiforme with an aim to unveil genetic changes that are common to both types of astrocytic tumours. Non-random allelic losses were identified on $9 p$ (67\%), 10p (62\%), I0q (76\%), I3q (60\%), I4q (50\%), and 17p (65\%). Allelic losses of 10p, 10q, I4q and 17p were common genetic alterations detectable in both fibrillary astrocytomas and glioblastoma multiforme. In addition, two common regions of deletions on chromosome 14 were mapped to 14q22.3-32.1 and 14q32.1-qter, suggesting the presence of two putative tumour suppressor genes. In conclusion, our comprehensive allelotype analysis has unveiled several critical tumour suppressor loci that are involved in the development of fibrillary astrocytomas and glioblastoma multiforme. Although these two types of brain tumours are believed to evolve from different genetic pathways, they do share some common genetic changes. Our results indicate that deletions of chromosome $14 q$ is a recurrent genetic event in the development of astrocytoma and highlight the subchromosomal regions on this chromosome that are likely to contain putative tumour suppressor genes involved in the oncogenesis of astrocytic tumours.

British Journal of Cancer (2002) 87, 218-224. doi:10.1038/sj.bjc.6600430 www.bjcancer.com

(c) 2002 Cancer Research UK

Keywords: fibrillary astrocytoma; glioblastoma multiforme; allelotyping; loss of heterozygosity

Diffusely infiltrating astrocytomas are the most frequent intracranial neoplasms and account for more than $60 \%$ of all primary brain tumours. According to the World Health Organization (WHO) classification, diffuse astrocytomas are graded into fibrillary astrocytoma (grade II), anaplastic astrocytoma (grade III) and glioblastoma multiforme (GBM; grade IV) (Kleihues et al, 2000). The fibrillary astrocytomas tumours have the inherent tendency for malignant progression to higher grade, with GBM as the most malignant phenotypic endpoint. Such glioblastomas are termed secondary GBM, in contrast to those de novo GBM that arise without prior known low-grade astrocytic tumours.

Molecular genetic studies on astrocytic tumours have been performed extensively to investigate the common events involved. In low-grade astrocytic tumours, chromosomal regions with frequent loss have been identified on $6 \mathrm{q}, 10 \mathrm{p}, 13 \mathrm{q}, 17 \mathrm{p}$ and $22 \mathrm{q}$ (Tsuzuki et al, 1996; Ino et al, 1999; Miyakawa et al, 2000). Mutation of the TP53 gene, which is located on 17p, is the most common genetic alteration detected in $>60 \%$ tumours (von Deimling et al, 1992a). Gains of chromosomal arms 7q and 8q

*Correspondence: H-k Ng; E-mail: hkng@cuhk.edu.hk

Received 28 January 2002; revised 23 April 2002; accepted 3 May 2002 are also detected in a subset of low-grade astrocytic tumours (Schrock et al, 1996; Nishizaki et al, 1998). Moreover, low-grade astrocytic tumours often recur with concomitant malignant progression to anaplastic astrocytomas or glioblastomas by acquiring additional genetic changes. These alterations include $19 \mathrm{q}$ loss, RB1 alteration, 10q loss, PTEN/MMAC1 mutations, loss of DCC expression, and overexpression of PDGFR- $\alpha$ (Hermanson et al, 1992; von Deimling et al, 1992b; Karlbom et al, 1993; Henson et al, 1994; Li et al, 1997; Reyes-Mugica et al, 1997; Steck et al, 1997). On the other hand, de novo GBM is characterised by a distinct set of genetic changes that involve gene amplification and overexpression of EGFR and MDM2, CDKN2A deletion, 10p and 10q loss, and RB1 alteration ( Libermann et al, 1985; Karlbom et al, 1993; Reifenberger et al, 1993; Schmidt et al, 1994; Ueki et al, 1996).

Molecular genetic studies on low-grade astrocytic tumours have been limited and these studies focused mostly on specific chromosomes and genes that are commonly affected in other tumour types. It still remains unknown whether other autosomes are involved in the development of astrocytomas. In this study, we performed a comprehensive genome-wide allelotype analysis on 17 low-grade fibrillary astrocytomas, using 382 microsatellite markers that cover the 22 autosomes, with an aim to detect critical 
tumour suppressor loci that are involved in astrocytoma formation. We also allelotyped 21 de novo GBM to identify genetic events that are common to both types of astrocytic tumours.

\section{MATERIALS AND METHODS}

\section{Specimens}

Seventeen fibrillary astrocytomas and 21 de novo GBM were collected from hospitals in Hong Kong and China. Tumours obtained from surgery were immediately stored at $-80^{\circ} \mathrm{C}$ until use. All tumours were diagnosed according to the recent WHO criteria (Kleihues et al, 2000). Each tumour was histologically confirmed to have neoplastic cell content greater than $80 \%$. The corresponding peripheral blood of each patient was also obtained as constitutional control. The mean age of patients with fibrillary astroytomas was $34 \pm 7.3$ years and the male/female ratio was 1.1, whereas patients with GBM tended to be older with a mean age of $45.2 \pm 14.5$ years and their male/female ratio was 0.9 . None of the cases were recurrences.

\section{DNA extraction}

About $20-30$ pieces of $10 \mu \mathrm{m}$ thick frozen sections of each tumour were cut for DNA extraction. High molecular weight genomic DNA from both blood and tumour was purified using conventional proteinase $\mathrm{K}$ digestion and phenol-chloroform extraction.

\section{Allelotype analysis}

A high-resolution genome-wide allelotype analysis was performed according to reported protocols (Tong et al, 2001). Briefly, 382 microsatellite loci derived from 22 autosomes were examined for allelic imbalances. The average interval of these loci is about $10 \mathrm{cM}$. The polymorphic microsatellite markers were obtained from the ABI Prism Linkage Mapping Set V.2 (Applied Biosystems, CA, USA) and were originally selected from the Généthon human linkage map. The set consists of primer pairs end-labelled with either one of three fluorochromes: FAM, HEX, or NED. PCR was performed in a final volume of $7.5 \mu \mathrm{l}$ containing two primer pairs (2.5 pmoles of each primer), $60 \mathrm{ng}$ of DNA, $10 \mathrm{~mm}$ Tris$\mathrm{HCl}(\mathrm{pH} 8.3), 50 \mathrm{mM} \mathrm{KCl}, 0.2 \mathrm{~mm}$ deoxyribonucleoside triphosphates, $2.5 \mathrm{mM} \mathrm{MgCl}_{2}$ and 0.6 unit of AmpliTaq Gold DNA polymerase (Applied Biosystems). To facilitate high throughput of samples, all liquid handling and thermal cycling were carried out in an ABI Prism 877 robotic workstation (Applied Biosystems). PCR was started, according to manufacturer's recommendation, with $95^{\circ} \mathrm{C}$ for $15 \mathrm{~min}$, followed by 10 cycles composed of $94^{\circ} \mathrm{C}$ for $15 \mathrm{~s}, 55^{\circ} \mathrm{C}$ for $15 \mathrm{~s}$ and $72^{\circ} \mathrm{C}$ for $30 \mathrm{~s}$, and another 22 cycles composed of $89^{\circ} \mathrm{C}$ for $15 \mathrm{~s}, 55^{\circ} \mathrm{C}$ for $15 \mathrm{~s}$ and $72^{\circ} \mathrm{C}$ for $30 \mathrm{~s}$. Amplified PCR products of multiple loci were pooled and electrophoresed in denaturing 5\% polyacrylamide gels on an ABI Prism 377 automated DNA sequencer (Applied Biosystems). The data collected were analysed using GeneScan Analysis software version 3.1 (Applied Biosystems). Allelic imbalance was defined by calculating the allelic ratio (AR) of both normal $(\mathrm{N})$ and tumour $(\mathrm{T})$ DNA, where AR was the ratio of peak height of the longer allele $(\mathrm{N} 2$ or $\mathrm{T} 2)$ to that of the shorter allele (N1 or T1), i.e., $\mathrm{AR}=(\mathrm{N} 2 / \mathrm{N} 1) /(\mathrm{T} 2 / \mathrm{T} 1)$. Allelic imbalance was indicated when the ratio was greater than 1.5 or smaller than 0.5 , representing loss of longer or shorter allele respectively.

\section{RESULTS}

To localise critical tumour suppressor loci involved in the tumorigenesis of astrocytoma, we performed a comprehensive genomewide allelotype analysis on 17 fibrillary astrocytomas and 21 GBM. All 22 autosomes were examined for allelic imbalances. An average of $70 \%$ informative loci/case was detected in our series. Allelic imbalances were seen in all 39 autosomal arms. Representative results of allelic imbalances at selected microsatellite loci are illustrated in Figure 1.

\section{Allelotype of fibrillary astrocytomas}

The frequency of allelic imbalances for individual chromosomal arm in the low-grade astrocytoma series varied from $0 \%(12 \mathrm{q}$, $16 \mathrm{p}, 20 \mathrm{q}$ and $22 \mathrm{q}$ ) to $53 \%$ (17p) (Figure 2). The mean percentage of allelic imbalances was $15 \pm 10 \%$. In the present study, $25 \%$ (mean percentage+one standard deviation) was chosen to be a significant percentage of allelic imbalances. This represents the 95\% confidence upper limit for the overall rate for random chromosome loss or imbalance in tumours. Non-random allelic imbalances above the baseline $(25 \%)$ were identified on chromosomal arms $10 \mathrm{p}(29 \%), 10 \mathrm{q}(29 \%), 14 \mathrm{q}(35 \%), 17 \mathrm{p}(53 \%)$, and $19 \mathrm{q}$ (29\%). Comparative genomic hybridisation (CGH) analysis was also performed on three tumours, in which sufficient DNA was available. These tumours were confirmed to have deletion regions identified by allelotyping (data not shown).

We have also delineated the common regions of deletion (CRDs) on chromosomes that are frequently lost in fibrillary astrocytomas. Two CRDs were mapped on chromosome 10, one was located to a region of $5.2 \mathrm{cM}$ on 10p14-15.1 (between D10S591 and D10S189) and the other was mapped to a region of $42.4 \mathrm{cM}$ between 10q25.1 (marker D10S597) and the telomere of long arm. The LOH frequency at this region was $29 \%$ (five of 17 informative cases). The CRD on chromosome $14 \mathrm{q}$ was localized to a region of $85 \mathrm{cM}$ between 14q21.2 (D14S288) and the 14q telomere (Figure 4). The LOH frequency at this region was 35\% (six of 17 informative cases). On chromosome 17, one CRD was defined within $17 \mathrm{p} 11.2$ (D17S799) and the $17 \mathrm{p}$ telomere, with a $\mathrm{LOH}$ frequency of $53 \%$ (nine of 17 informative cases). Another CRD of $51.2 \mathrm{cM}$ was delineated on chromosome 19q12-13.4, between markers D19S414 and D19S210. The LOH frequency at this region was $29 \%$ (five of 17 informative cases). In addition, chromosomal arms with frequencies of allelic imbalances higher than the mean percentage of $\mathrm{LOH}$ were identified on $2 \mathrm{q}(24 \%), 3 \mathrm{p}(18 \%), 6 \mathrm{p}$ $(18 \%), 6 q(24 \%), 7 q(18 \%), 8 q(18 \%), 10 p(24 \%), 11 p(18 \%)$, $13 \mathrm{q}(18 \%), 15 \mathrm{q}(18 \%)$ and $21 \mathrm{q}(18 \%)$.

\section{Allelotype of GBM}

The frequency of allelic imbalances for individual chromosomal arm in the GBM series varied from $10 \%$ (8q) to $76 \%$ (10q) (Figure 3 ). The mean percentage of allelic imbalance was $32 \pm 15.9 \%$, with $47.9 \%$ (mean percentage+one standard deviation) chosen to be a significant percentage of allelic imbalances. Non-random allelic

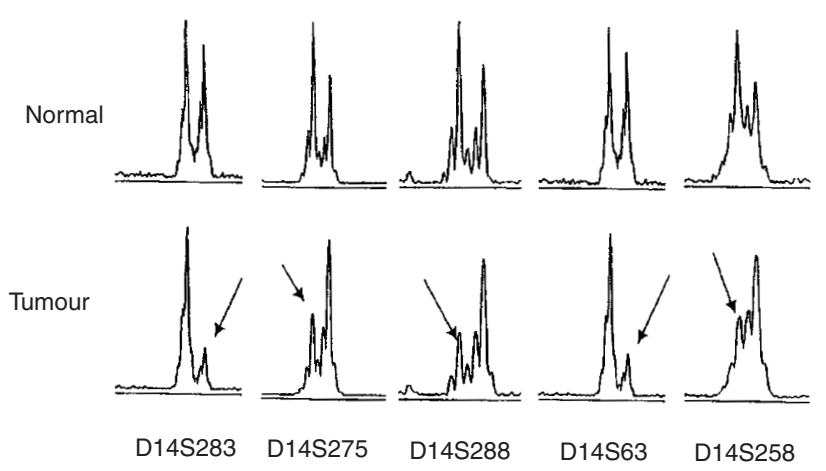

Figure I Representative results of allelotype analysis. Allelic patterns of five polymorphic loci on chromosomal arm 14q examined in a fibrillary astrocytoma (case $2 \mathrm{l}$ ) are shown. Allelic loss is indicated by arrow. 


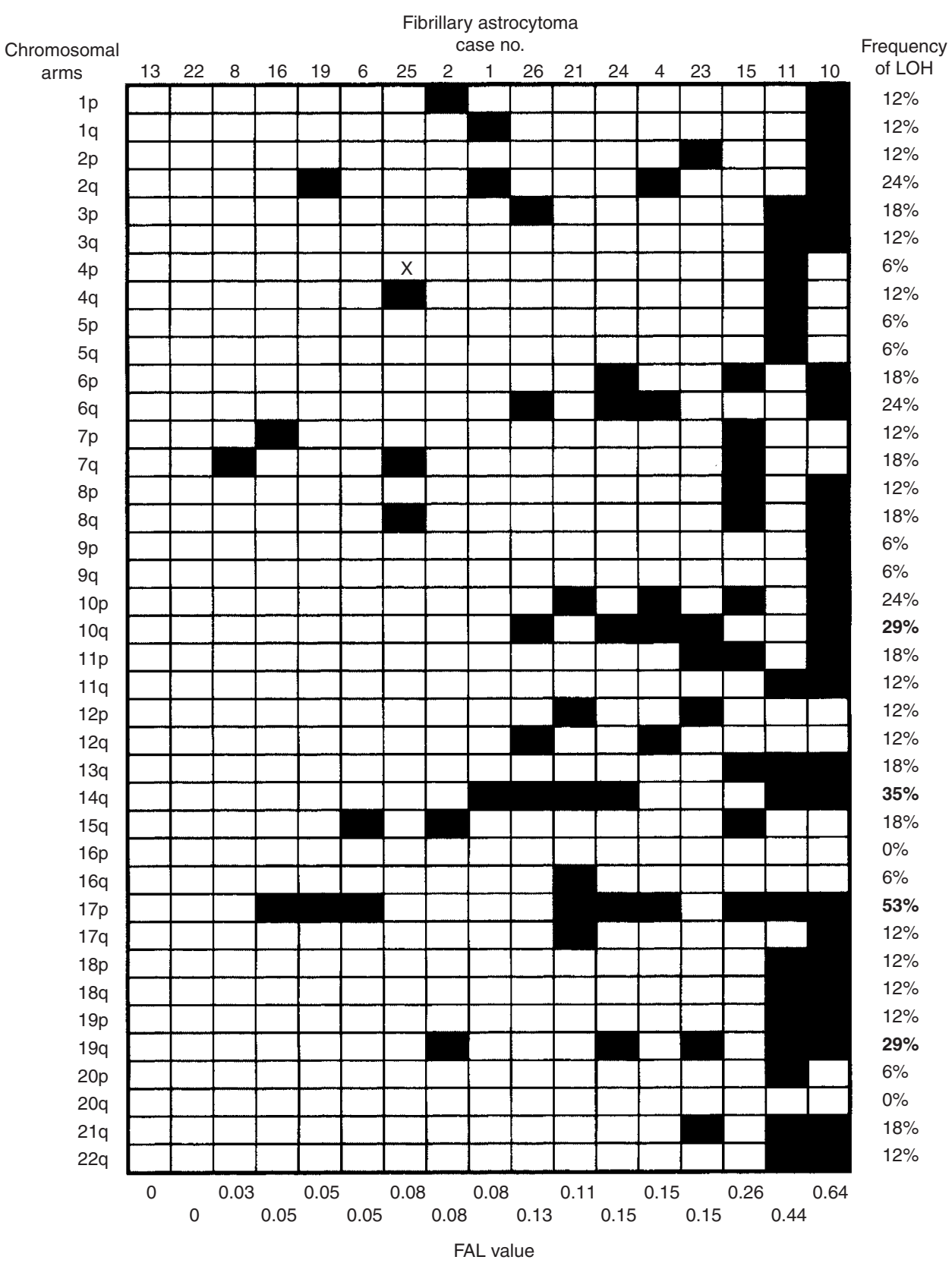

Figure 2 Summary of allelic imbalances detected in 17 fibrillary astrocytoma. Case number is indicated on top and fractional allelic loss (FAL) value is shown at bottom. Frequency of $\mathrm{LOH}$ is indicated on right, with bold number representing non-random allelic imbalance frequency above the baseline level (25\%). Filled box represents allelic imbalance detected in specified chromosomal arm and open box indicate chromosomal arm with no detectable allelic imbalance. X, not done.

imbalances were detected on chromosomes $9 \mathrm{p}(67 \%), 10 \mathrm{p}(62 \%)$, $10 \mathrm{q}(76 \%), 13 \mathrm{q}(60 \%), 14 \mathrm{q}(50 \%)$ and $17 \mathrm{p}(65 \%)$.

The CRD of $11.1 \mathrm{cM}$ on chromosome 9 was mapped to $9 \mathrm{p} 21-23$, flanked by markers D9S286 and D9S285. The LOH frequency at this region was $62 \%$. Three CDRs were observed on chromosome 10 , at 10p15-pter (16.5 cM between D10S189 and D10S249), 10q23.3-25.1 (9.9 cM flanked by D10S185 and D10S597), and 10q25.3-26.3 (30.6 cM between D10S1693 and D10S1651). The $\mathrm{LOH}$ frequencies at these deletion regions were $58 \%$ (11 of 19 informative cases), 77\% (13 out of 17) and 58\% (11 out of 19), respectively. On chromosome 13 , the CDR lied in a region of $50.7 \mathrm{cM}$, between markers D13S217 and D13S170. The frequency of $\mathrm{LOH}$ at this $13 \mathrm{q} 13.2-31$ deletion region was $50 \%$ (10 out of
20). Two CRDs were located on chromosome 14: one was mapped to $14 \mathrm{q} 22.3-32.1$ (48.9 cM between D14S276 and D14S280) and the other was localised between $14 \mathrm{q} 32.1$ and $14 \mathrm{q}$ telomere $(28.3 \mathrm{cM}$ between D14S280 and pter), with LOH frequency of 50\% (10 out of 20 ) and $44 \%$ (eight out of 18), respectively (Figure 4). Another CRD was observed between 17p12 (D17S1852) and 17p telomere $(23 \mathrm{cM})$. This deletion region was detected in $60 \%$ (12 out of 20 ) of informative cases.

CGH analysis was performed on nine GBM and confirmed the deletion regions identified by allelotyping. Chromosomal regions that show gain of genetic material had been analysed by arraybased $\mathrm{CGH}$, in which 58 oncogenes/amplicons were investigated, and were reported elsewhere (Hui et al, 2001). 


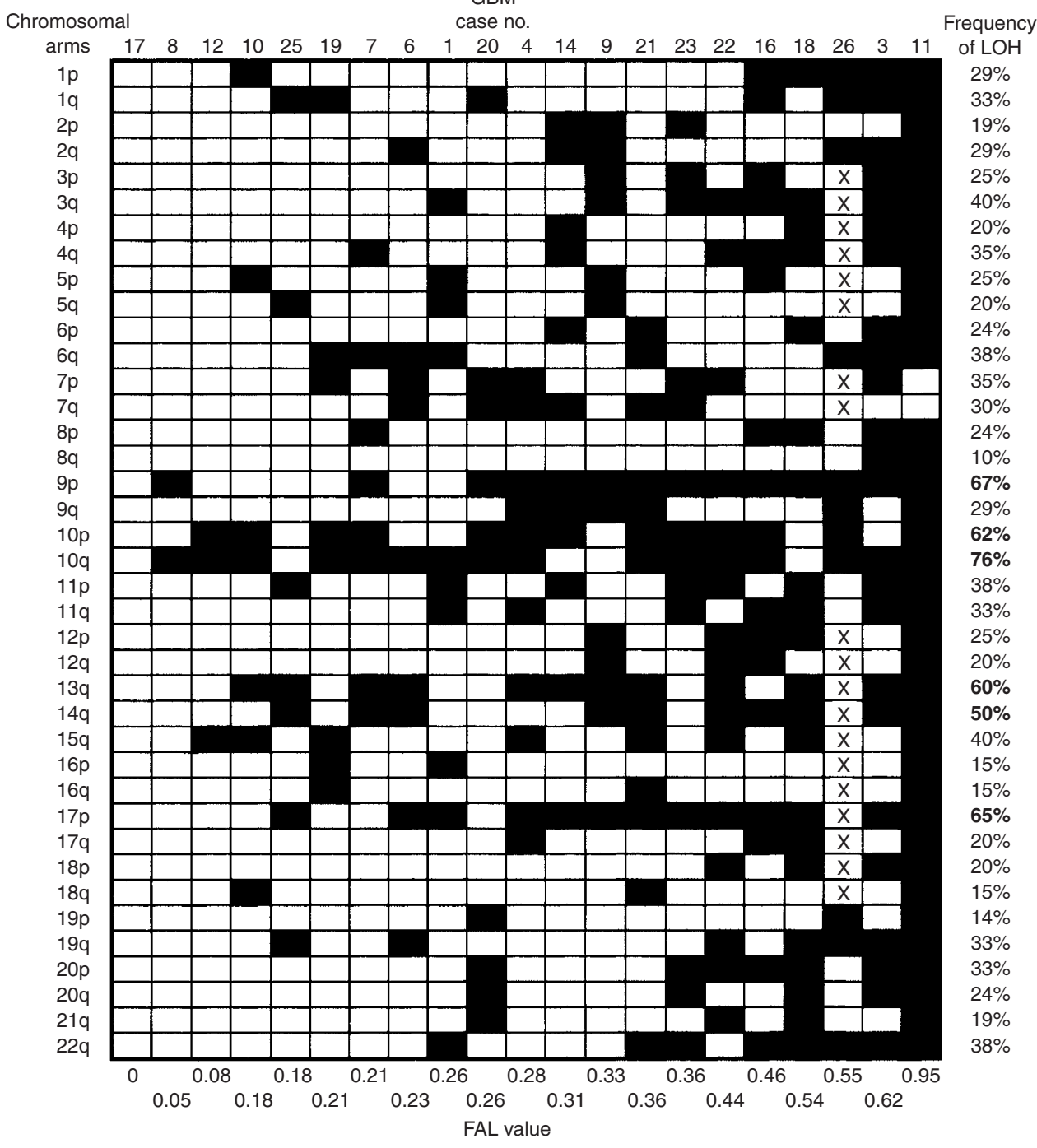

Figure 3 Summary of allelic imbalances detected in 21 GBM. Case number is indicated on top and fractional allelic loss (FAL) value is shown at bottom. Frequency of $\mathrm{LOH}$ is indicated on right, with bold number representing non-random allelic imbalance frequency above the baseline level (47.9\%). Filled box represents allelic imbalance detected in specified chromosomal arm and open box indicate chromosomal arm with no detectable allelic imbalance. X, not done.

\section{Fractional allelic loss}

The fractional allelic loss (FAL) for each tumour was also determined (Figures 2 and 3). FAL is defined as the fraction of the total number of informative chromosomal arms that displays allelic loss (Vogelstein et al, 1989). In the present study, the mean FAL values for fibrillary astrocytomas and GBM were 0.15 (range, $0-$ 0.67 ) and 0.33 (range, $0-0.95$ ), respectively. This result indicates that additional genetic alterations were accumulated in GBM. There was no association between the FAL values and the age/sex of the patients. By using Mann-Whitney $U$-test, high FAL value was found to be significantly associated with chromosomes $10 \mathrm{q}$ $(P=0.05), 14 \mathrm{q}(P=0.01)$ and $19 \mathrm{q}(P=0.01)$ in fibrillary astrocytomas, and $9 \mathrm{p}(P<0.01), 14 \mathrm{q}(P=0.02)$ and $17 \mathrm{p}(P<0.01)$ in GBM. Moreover, microsatellite instability was rarely found in our series.

\section{DISCUSSION}

In this study, we performed a comprehensive genome-wide allelotype analysis on a series of 17 low-grade astrocytomas and 21 de novo GBM with an aim to localise putative tumour suppressor loci involved in astrocytic tumours. Non-random chromosome losses were identified on $10 \mathrm{p}, 10 \mathrm{q}, 14 \mathrm{q}, 17 \mathrm{p}$ and $19 \mathrm{q}$ in fibrillary astrocytomas, and on $9 p, 10 p, 10 q, 13 q, 14 q$ and $17 p$ in GBM (Table 1). These results strongly indicate that alterations of these chromosomes play critical roles in the genesis of astrocytoma.

Mutation of the TP53 gene and/or allelic loss of $17 \mathrm{p}$ is the most prominent genetic alteration detected in $>60 \%$ of fibrillary astrocytomas (von Deimling et al, 1992a). Study on malignant progression of low-grade astrocytomas to high-grade tumours revealed that the frequencies of TP53 mutation were comparable in both groups of tumours, suggesting that TP53 mutation is an early event in the evolution of diffuse astrocytoma (Watanabe et al, 1997). Our allelotype analysis identifies a CRD between $17 \mathrm{p} 11.2$ and $17 \mathrm{p}$ telomere, which encompasses the TP53 locus, as the major genetic alteration in the low-grade astrocytomas.

In the present study, allelic loss of $14 \mathrm{q}$ is the second most common genetic alteration detected in fibrillary astrocytomas. Since chromosome 14 has never been a target under investigation for genetic changes in low-grade astrocytomas, we therefore 

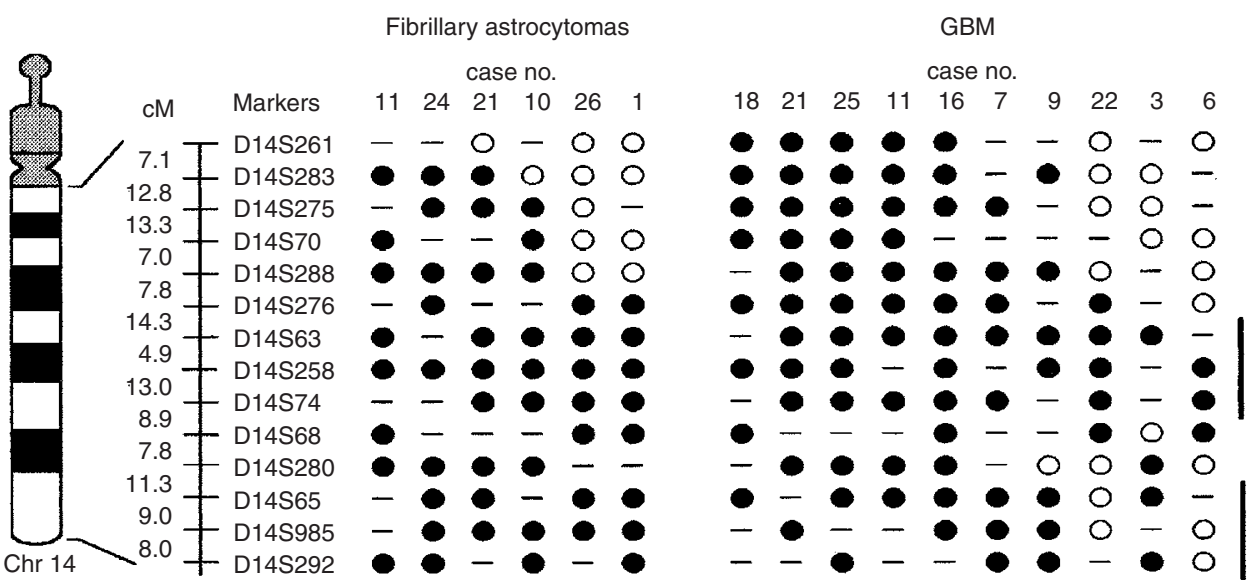

Figure 4 Delineation of common region of deletion on chromosomal arm 14q in astrocytic tumors. Fourteen polymorphic loci, with their respective genetic intervals in centiMorgan (cM), were examined for allelic loss. Two common regions of deletion (thick bars) are identified: $14 \mathrm{q} 22.3-32.1$ and 14q32. I-qter. Filled circle represents loss of heterozygosity and open circle denotes retention of heterozygosity. Dash line indicates homozygosity. The candidate tumour suppressor gene, MLH3, is located between markers DI4S258 and DI4S74 on the genetic map.

Table I Summary of critical deletion regions identified in 38 astrocytic tumours

\begin{tabular}{|c|c|c|c|}
\hline \multirow[b]{2}{*}{$\begin{array}{l}\text { Chromosomal } \\
\text { arms }\end{array}$} & \multicolumn{2}{|c|}{ Frequency of LOH (\%) } & \multirow[b]{2}{*}{$\begin{array}{l}\text { Deletion } \\
\text { regions }\end{array}$} \\
\hline & $\begin{array}{c}\text { Fibrillary } \\
\text { astrocytoma }\end{array}$ & GBM & \\
\hline $9 p$ & 6 & 62 & $9 p 21-23$ \\
\hline $10 p$ & 29 & 58 & IOp|5-pter \\
\hline $10 q$ & 29 & $77 / 58$ & I0q23.3-24.1// 0q25.3-26.3 \\
\hline $13 q$ & 18 & 60 & $13 q|3.2-3|$ \\
\hline $14 \mathrm{q}$ & 35 & $50 / 40$ & I 4q22.3-32.1/14q32.1 -qter \\
\hline $17 \mathrm{q}$ & 53 & 60 & 17q | 1.2-pter \\
\hline $19 q$ & 29 & 33 & $19 q 12-13.4$ \\
\hline
\end{tabular}

${ }^{a}$ Bold number is $\mathrm{LOH}$ frequency considered to be significant.

reviewed genetic results from three genome-wide studies covering a total of 40 fibrillary astrocytomas (Schrock et al, 1996; Sallinen et al, 1997; von Deimling et al, 2000). Sallinen et al (1997) detected chromosome loss of $14 \mathrm{q}$ in $18 \%$ tumours by CGH, whereas Schrock et al (1996) (with CGH) and von Deimling et al (2000) (with allelotype analysis) detected no alteration in chromosome $14 \mathrm{q}$. It should be noted that only three polymorphic markers on chromosome $14 \mathrm{q}$ were examined in the latter allelotyping study. Our allelotype analysis investigated 14 polymorphic loci on the long arm of chromosome 14 and detected $35 \%$ of tumours with $14 \mathrm{q}$ loss. Moreover, we were able to delineate a CRD between $14 \mathrm{q} 21.2$ and $14 \mathrm{q}$ telomere. Although low-grade astrocytomas and de novo GBM are regarded as two groups of brain tumours with distinct genetic changes, we did observe $14 \mathrm{q}$ loss in $50 \%$ of GBM in our series. In addition, two CRDs were localised on chromosome 14 in GBM, one mapped to 14q22.332.1 and the other defined within $14 \mathrm{q} 32.1$ and $14 \mathrm{q}$ telomere (Figure 4). The presence of two CRDs suggests that there are two tumour suppressor genes located on chromosome 14q. It seems that chromosome $14 \mathrm{q}$ loss is a common event for both low-grade astrocytomas and de novo GBM. Further study is needed to refine the deletion regions on chromosome $14 \mathrm{q}$ for positional cloning of the tumour suppressor genes involved in astrocytomas.

Frequent deletion of $14 \mathrm{q}$ has also been detected in a variety of tumours such as meningioma, neuroblastoma, breast and ovarian cancer (Suzuki et al, 1989; Bandera et al, 1997; Tse et al, 1997; O'Connell et al, 1999). Deletion mapping has identified at least three tumour suppressor loci on $14 \mathrm{q}$ that are important for cancer development. These regions are mapped to $14 \mathrm{q} 11-13,14 \mathrm{q} 22-24$ and 14q32 (Bandera et al, 1997; Tse et al, 1997; O'Connell et al, 1999). The latter two regions overlap with the deletion regions delineated in this study. Thus, deletion at $14 \mathrm{q}$ is a common feature in different tumour types including astrocytic tumours. A candidate tumour suppressor gene, MLH3, has been identified on 14q24.3. MLH3 is a mismatch-repair gene and mutation of this gene is associated with microsatellite instability in colorectal tumours (Lipkin et al, 2001). Other mismatch-repair genes such as MLH1 and MSH2 have been found to be mutated in a subset of malignant gliomas (Leung et al, 1998). Whether genetic alteration of MLH3 plays a role in astrocytoma formation remains to be determined.

Allelic loss of chromosome 19q was detected in $29 \%$ and $33 \%$ of fibrillary astrocytomas and GBM, respectively, in our allelotype analysis. These results are consistent with previous allelotyping study and suggest the presence of a tumour suppressor gene on $19 \mathrm{q}$. Chromosome $19 \mathrm{q}$ loss is the only known genetic alteration that is shared by the three major glioma subtypes, astrocytomas, oligodendrogliomas and oligoastrocytomas (von Deimling et al, 1992b). The deletion breakpoint on $19 \mathrm{q}$ has been mapped to the segment $19 q 13.3$. Recent finer deletion mapping have identified two overlapping regions, flanked by markers D19S241 and STD, with high frequency of allelic loss (Rosenberg et al, 1996; Smith et al, 2000a). A partial transcript map has been constructed for this deletion region (Smith et al, 2000b) and it remains to be determined if any of these genes is the tumour suppressor for gliomas.

Deletions of chromosome 10 are among the most frequent genetic alterations in astrocytomas, particularly in the high-grade variants. Several deletion regions have been identified on both arms of chromosome 10 and these regions have been mapped to $10 \mathrm{p} 15$, 10p14 and 10q25 (Rasheed et al, 1995; Voesten et al, 1997; Ichimura et al, 1998; Kon et al, 1998). The presence of tumour suppressor genes on these deletion regions is supported by chromosome transfer studies (Pershouse et al, 1993; Kon et al, 1998). Two putative tumour suppressor genes, namely PTEN/MMAC1 and DMBT1, have been identified on chromosome 10q23.3 and 10q25, respectively (Li et al, 1997; Mollenhauer et al, 1997; Steck et al, 1997). In this study, we detected chromosome 10 loss in seven (41\%) fibrillary astrocytomas and 18 (86\%) GBM, supporting the notion that chromosome 10 plays an important role in astrocytoma formation. Recent study also suggests that low-grade astrocytomas with chromosome 10 loss are likely to undergo malignant progression (Ichimura et al, 1998). Whether the fibril- 
lary astroytomas examined in this study behave similarly requires further follow-up evaluation.

In the GBM series, we detected non-random chromosome losses on 9p13.3-21.3 (58\%), 10p15-pter (77\%), 10q23.3-25.1 (58\%), $13 q 13.2-31$ (50\%), 14q22.3-32.1 (50\%) and 17p12-pter (60\%). We compared our results with the published allelotyping data (von Deimling et al, 2000) and found that the data, except for $\mathrm{LOH} 17 \mathrm{q}$, from both studies were largely comparable. The frequency of $17 \mathrm{q}$ loss in de novo GBM is the highest frequency reported to date and this is consistent with another series of GBM that we examined in previous study (Cheng et al, 1999). The putative tumour suppressor genes on some of these chromosomes have been identified and their associated genetic pathways are elucidated. For instance, CDKN2A (on 9p21) and RB1 (on $13 \mathrm{q} 14.2)$ are involved in the cell cycle control; p14 ${ }^{\mathrm{ARF}}$ (on 9p21) negatively regulates TP53 (on 17p13.1) function; and PTEN/ MMAC1 (on 10q23.3) is involved in the PI3K/AKT pathway. These pathways are believed to play important roles in the development and progression of astrocytoma. Recently, Pieper's group using a functional approach demonstrated that normal astrocytes could be transformed to astrocytoma by interfering with the telomerase, RAS, CDKN2A/RB1 and TP53 pathways (Sonoda et al, 2001a). They further showed that additional disturbance of the PI3K/ AKT pathway elicited a glioblastoma phenotype in the in vitro transformed astrocytoma (Sonoda et al, 2001b). Thus, conversion of normal astrocytes to glioblastoma requires disruptions of at least five genetic pathways. The roles of other tumour suppressor loci on $10 \mathrm{p}$ and $14 \mathrm{q}$ in astrocytoma formation remain to be elucidated.

In conclusion, our comprehensive allelotype analysis have unveiled several critical tumour suppressor loci that are involved in the development of fibrillary astrocytomas and GBM. Although these two types of brain tumours are believed to evolve from different genetic pathways, they do share some common genetic changes. Our results indicate that deletions of chromosome $14 \mathrm{q}$ is a recurrent genetic event in the development of astrocytoma and highlight the subchromosomal regions on this chromosome that are likely to contain putative tumour suppressor genes involved in the oncogenesis of astrocytic tumours.

\section{REFERENCES}

Bandera CA, Takahashi H, Behbakht K, Liu PC, LiVolsi VA, Benjamin I, Morgan MA, King SA, Rubin SC, Boyd J (1997) Deletion mapping of two potential chromosome 14 tumor suppressor gene loci in ovarian carcinoma. Cancer Res 57: 513-515

Cheng Y, Ng HK, Ding M, Zhang SF, Pang JC, Lo KW (1999) Molecular analysis of microdissected de novo glioblastomas and paired astrocytic tumors. J Neuropathol Exp Neurol 58: 120-128

Henson JW, Schnitker BL, Correa KM, von Deimling A, Fassbender F, Xu HJ, Benedict WF, Yandell DW, Louis DN (1994) The retinoblastoma gene is involved in malignant progression of astrocytomas. Ann Neurol 36: $714-721$

Hermanson M, Funa K, Hartman M, Claesson-Welsh L, Heldin CH, Westermark B, Nister M (1992) Platelet-derived growth factor and its receptors in human glioma tissue: expression of messenger RNA and protein suggests the presence of autocrine and paracrine loops. Cancer Res 52: 3213-3219

Hui AB, Lo KW, Yin XL, Poon WS, Ng HK (2001) Detection of multiple gene amplifications in glioblastoma multiforme using array-based comparative genomic hybridization. Lab Invest 81: 717-723

Ichimura K, Schmidt EE, Miyakawa A, Goike HM, Collins VP (1998) Distinct patterns of deletion on 10p and 10q suggest involvement of multiple tumor suppressor genes in the development of astrocytic gliomas of different malignancy grades. Genes Chrom Cancer 22: 9-15

Ino Y, Silver JS, Blazejewski L, Nishikawa R, Matsutani M, von Deimling A, Louis DN (1999) Common regions of deletion on chromosome 22q12.3q13.1 and 22q13.2 in human astrocytomas appear related to malignancy grade. J Neuropathol Exp Neurol 58: 881-885

Kleihues P, Davis RL, Ohgaki H, Burger PC, Westphal MM, Cavenee WK (2000) In Pathology and Genetics of Tumours of the Nervous System, Kleihues P, Cavenee WK (eds) pp $22-39$. Lyon: IARC Press

Kon H, Sonoda Y, Kumabe T, Yoshimoto T, Sekiya T, Murakami Y (1998) Structural and functional evidence for the presence of tumor suppressor genes on the short arm of chromosome 10 in human gliomas. Oncogene 16: $257-263$

Karlbom AE, James CD, Boethius J, Cavenee WK, Collins VP, Nordenskjold M, Larsson C (1993) Loss of heterozygosity in malignant gliomas involves at least three distinct regions on chromosome 10. Hum Genet 92: 169-174

Leung SY, Chan TL, Chung LP, Chan AS, Fan YW, Hung KN, Kwong WK, Ho JW, Yuen ST (1998) Microsatellite instability and mutation of DNA mismatch repair genes in gliomas. Am J Pathol 153: 1181-1188

Li J, Yen C, Liaw D, Podsypanina K, Bose S, Wang SI, Puc J, Miliaresis C, Rodgers L, McCombie R, Bigner SH, Giovanella BC, Ittmann M, Tycko B, Hibshoosh H, Wigler MH, Parsons R (1997) PTEN, a putative protein tyrosine phosphatase gene mutated in human brain, breast, and prostate cancer. Science 275: $1943-1947$

Libermann TA, Nusbaum HR, Razon N, Kris R, Lax I, Soreq H, Whittle N, Waterfield MD, Ullrich A, Schlessinger J (1985) Amplification, enhanced expression and possible rearrangement of EGF receptor gene in primary human brain tumours of glial origin. Nature 313: 144-147
Lipkin SM, Wang V, Stoler DL, Anderson GR, Kirsch I, Hadley D, Lynch HT, Collins FS (2001) Germline and somatic mutation analyses in the DNA mismatch repair gene MLH3: Evidence for somatic mutation in colorectal cancers. Hum Mutat 17: 389-396

Miyakawa A, Ichimura K, Schmidt EE, Varmeh-Ziaie S, Collins VP (2000) Multiple deleted regions on the long arm of chromosome 6 in astrocytic tumours. Br J Cancer 82: $543-549$

Mollenhauer J, Wiemann S, Scheurlen W, Korn B, Hayashi Y, Wilgenbus KK, von Deimling A, Poustka A (1997) DMBT1, a new member of the SRCR superfamily, on chromosome 10q25.3-26.1 is deleted in malignant brain tumours. Nat Genet 17: $32-39$

Nishizaki T, Ozaki S, Harada K, Ito H, Arai H, Beppu T, Sasaki K (1998) Investigation of genetic alterations associated with the grade of astrocytic tumor by comparative genomic hybridization. Genes Chrom Cancer 21: $340-346$

O'Connell P, Fischbach K, Hilsenbeck S, Mohsin SK, Fuqua SA, Clark GM, Osborne CK, Allred DC (1999) Loss of heterozygosity at D14S62 and metastatic potential of breast cancer. J Natl Cancer Inst 91: 1391 - 1397

Pershouse MA, Stubblefield E, Hadi A, Killary AM, Yung WK, Steck PA (1993) Analysis of the functional role of chromosome 10 loss in human glioblastomas. Cancer Res 53: 5043-5050

Rasheed BK, McLendon RE, Friedman HS, Friedman AH, Fuchs HE, Bigner DD, Bigner SH (1995) Chromosome 10 deletion mapping in human gliomas: a common deletion region in 10q25. Oncogene 10: 2243-2246

Reifenberger G, Liu L, Ichimura K, Schmidt EE, Collins VP (1993) Amplification and overexpression of the MDM2 gene in a subset of human malignant gliomas without p53 mutations. Cancer Res 53: 2736-2739

Reyes-Mugica M, Rieger-Christ K, Ohgaki H, Ekstrand BC, Helie M, Kleinman G, Yahanda A, Fearon ER, Kleihues P, Reale MA (1997) Loss of DCC expression and glioma progression. Cancer Res 57: 382-386

Rosenberg JE, Lisle DK, Burwick JA, Ueki K, von Deimling A, Mohrenweiser HW, Louis DN (1996) Refined deletion mapping of the chromosome 19q glioma tumor suppressor gene to the D19S412-STD interval. Oncogene 13: $2483-2485$

Sallinen SL, Sallinen P, Haapasalo H, Kononen J, Karhu R, Helen P, Isola J (1997) Accumulation of genetic changes is associated with poor prognosis in grade II astrocytomas. Am J Pathol 151: 1799-1807

Schmidt EE, Ichimura K, Reifenberger G, Collins VP (1994) CDKN2 (p16/ MTS1) gene deletion or CDK4 amplification occurs in the majority of glioblastomas. Cancer Res 54: 6321-6324

Schrock E, Blume C, Meffert MC, du Manoir S, Bersch W, Kiessling M, Lozanowa T, Thiel G, Witkowski R, Ried T, Cremer T (1996) Recurrent gain of chromosome arm $7 \mathrm{q}$ in low-grade astrocytic tumors studied by comparative genomic hybridization. Genes Chrom Cancer 15: 199-205 
Smith JS, Tachibana I, Lee HK, Qian J, Pohl U, Mohrenweiser HW, Borell TJ, Hosek SM, Soderberg CL, von Deimling A, Perry A, Scheithauer BW, Louis DN, Jenkins RB (2000a) Mapping of the chromosome 19 q-arm glioma tumor suppressor gene using fluorescence in situ hybridization and novel microsatellite markers. Genes Chrom Cancer 29: 16-25

Smith JS, Tachibana I, Pohl U, Lee HK, Thanarajasingam U, Portier BP, Ueki K, Ramaswamy S, Billings SJ, Mohrenweiser HW, Louis DN, Jenkins RB (2000b) A transcript map of the chromosome 19q-arm glioma tumor suppressor region. Genomics 64: $44-50$

Sonoda Y, Ozawa T, Hirose Y, Aldape KD, McMahon M, Berger MS, Piepe RO (2001a) Formation of intracranial tumors by genetically modified human astrocytes defines four pathways critical in the development of human anaplastic astrocytoma. Cancer Res 61: 4956-4960

Sonoda Y, Ozawa T, Aldape KD, Deen DF, Berger MS, Pieper RO (2001b) Akt pathway activation converts anaplastic astrocytoma to glioblastoma multiforme in a human astrocyte model of glioma. Cancer Res 61: $6674-6678$

Steck PA, Pershouse MA, Jasser SA, Yung WK, Lin H, Ligon AH, Langford LA, Baumgard ML, Hattier T, Davis T, Frye C, Hu R, Swedlund B, Teng DH, Tavtigian SV (1997) Identification of a candidate tumour suppressor gene, MMAC1, at chromosome 10q23.3 that is mutated in multiple advanced cancers. Nat Genet 15: $356-362$

Suzuki T, Yokota J, Mugishima H, Okabe I, Ookuni M, Sugimura T, Terada M (1989) Frequent loss of heterozygosity on chromosome 14q in neuroblastoma. Cancer Res 49: 1095-1098

Tong CY, Zheng PP, Pang JC, Poon WS, Chang AR, Ng HK (2001) Identification of novel regions of allelic loss in ependymomas by high-resolution allelotyping with 384 microsatellite markers. J Neurosurg 95: 9-14

Tse JY, Ng HK, Lau KM, Lo KW, Poon WS, Huang DP (1997) Loss of heterozygosity of chromosome $14 \mathrm{q}$ in low- and high-grade meningiomas. Hum Pathol 28: $779-785$

Tsuzuki T, Tsunoda S, Sakaki T, Konishi N, Hiasa Y, Nakamura M (1996) Alterations of retinoblastoma, p53, p16(CDKN2), and p15 genes in human astrocytomas. Cancer 78: $287-293$

Ueki K, Ono Y, Henson JW, Efird JT, von Deimling A, Louis DN (1996) CDKN2/p16 or RB alterations occur in the majority of glioblastomas and are inversely correlated. Cancer Res 56: 150-153

Voesten AM, Bijleveld EH, Westerveld A, Hulsebos TJ (1997) Fine mapping of a region of common deletion on chromosome arm 10p in human glioma. Genes Chrom Cancer 20: $167-172$

Vogelstein B, Fearon ER, Kern SE, Hamilton SR, Preisinger AC, Nakamura Y, White R (1989) Allelotype of colorectal carcinomas. Science 244: 207-211 von Deimling A, Eibl RH, Ohgaki H, Louis DN, von Ammon K, Petersen I, Kleihues P, Chung RY, Wiestler OD, Seizinger BR (1992a) p53 mutations are associated with $17 \mathrm{p}$ allelic loss in grade II and grade III astrocytoma. Cancer Res 52: $2987-2990$

von Deimling A, Louis DN, von Ammon K, Petersen I, Wiestler OD, Seizinger BR (1992b) Evidence for a tumor suppressor gene on chromosome 19q associated with human astrocytomas, oligodendrogliomas, and mixed gliomas. Cancer Res 52: 4277 - 4279

von Deimling A, Fimmers R, Schmidt MC, Bender B, Fassbender F, Nagel J, Jahnke R, Kaskel P, Duerr EM, Koopmann J, Maintz D, Steinbeck S, Wick W, Platten M, Muller DJ, Przkora R, Waha A, Blumcke B, Wellenreuther R, Meyer-Puttlitz B, Schmidt O, Mollenhauer J, Poustka A, Stangl AP, Lenartz D, von Ammon K (2000) Comprehensive allelotype and genetic analysis of 466 human nervous system tumors. J Neuropathol Exp Neurol 59: $544-558$

Watanabe K, Sato K, Biernat W, Tachibana O, von Ammon K, Ogata N, Yonekawa Y, Kleihues P, Ohgaki H (1997) Incidence and timing of p53 mutations during astrocytoma progression in patients with multiple biopsies. Clin Cancer Res 3: 523-530 